PROCEEDINGS OF THE

AMERICAN MATHEMATICAL SOCIETY

Volume 139, Number 12, December 2011, Pages 4255-4264

S 0002-9939(2011)10959-6

Article electronically published on April 29, 2011

\title{
GROUPS OF BANDED MATRICES WITH BANDED INVERSES
}

\author{
GILBERT STRANG
}

(Communicated by Birge Huisgen-Zimmermann)

\begin{abstract}
A product $A=F_{1} \ldots F_{N}$ of invertible block-diagonal matrices will be banded with a banded inverse: $A_{i} j=0$ and also $\left(A^{-1}\right)_{i j}=0$ for $|i-j|>w$. We establish this factorization with the number $N$ controlled by the bandwidths $w$ and not by the matrix size $n$. When $A$ is an orthogonal matrix, or a permutation, or banded plus finite rank, the factors $F_{i}$ have $w=1$ and we find generators of that corresponding group. In the case of infinite matrices, the $A=L P U$ factorization is now established but conjectures remain open.
\end{abstract}

\section{INTRODUCTION}

Banded matrices with banded inverses are unusual, but these exceptional matrices do exist. Block diagonal matrices are the first examples. Products of block diagonal matrices give many more examples (including useful ones). The main theorem in an earlier paper [10] is that all examples are produced this way, from multiplying invertible block diagonal matrices of bandwidth 1 .

When $A$ and $B$ are banded with banded inverses, $A^{-1}$ and $A B$ also have those properties. This group of matrices is all of $\operatorname{GL}(n)$ in the finite case (every matrix has bandwidth less than $n$ ). For singly infinite matrices this appears to be a new group. In both cases the key point of the theorem is that the number $N$ of block diagonal factors is controlled by the bandwidth $w$ and not by the matrix size $n$.

Theorem 1.1. The factors in $A=F_{1} F_{2} \ldots F_{N}$ can be chosen to be block diagonal, with 2 by 2 and 1 by 1 blocks. Then each generator $F$ and $F^{-1}$ has bandwidth $\leq 1$.

Here $w$ is the larger of the bandwidths of $A$ and $A^{-1}$. So $A_{i j}=0$ and $\left(A^{-1}\right)_{i j}=0$ for $|i-j|>w$. The number of factors $F$ could be as large as $C w^{2}$ (just to carry out ordinary elimination), but $N$ does not increase with $n$.

Important banded matrices with banded inverses arise in constructing orthogonal polynomials on the unit circle [5]. They also yield filter banks with perfect reconstruction, the key to wavelets. Those are block Toeplitz matrices in the wavelet case, and "CMV matrices" in other cases. Our earlier paper [10 applied an observation of Ilya Spitkovsky to separate these matrices into the minimum number $N=w$ of factors $F$ (each with bandwidth 1 ). We believe that these special (and short) factorizations can lead to useful algorithms.

Received by the editors October 22, 2010.

2010 Mathematics Subject Classification. Primary 15A23.

Key words and phrases. Banded matrix, banded inverse, Bruhat permutation, factorization, group generators, shifting index.

(C)2011 American Mathematical Society Reverts to public domain 28 years from publication 
For other $A$, the main step of the proof is to reach $A=B C$, two factors with diagonal blocks of size $2 w$. One of those factors begins with a block of size $w$, and it is this "offset" between the block positions in $B$ and $C$ that makes the proof work.

The form of this "offset product" is important even for $w=1$ :

$$
B C=\left[\begin{array}{llllll}
1 & & & & & \\
& 2 & 3 & & & \\
& 4 & 5 & & & \\
& & & 6 & 7 & \\
& & & 8 & 9 & \\
& & & & & 10
\end{array}\right]\left[\begin{array}{rrrrrr}
1 & 2 & & & & \\
3 & 4 & & & & \\
& & 5 & 6 & & \\
& 7 & 8 & & \\
& & & & 9 & 10 \\
& & & & 11 & 12
\end{array}\right] .
$$

The second row of $B C$ has 2 times [ $\left.\begin{array}{ll}3 & 4\end{array}\right]$ followed by 3 times [ $\left.\begin{array}{ll}5 & 6\end{array}\right]$. The third row of $B C$ has 4 times [ $\left.\begin{array}{ll}3 & 4\end{array}\right]$ followed by 5 times [ 56 6]. A pair of singular matrices lies side by side in the product $B C$ :

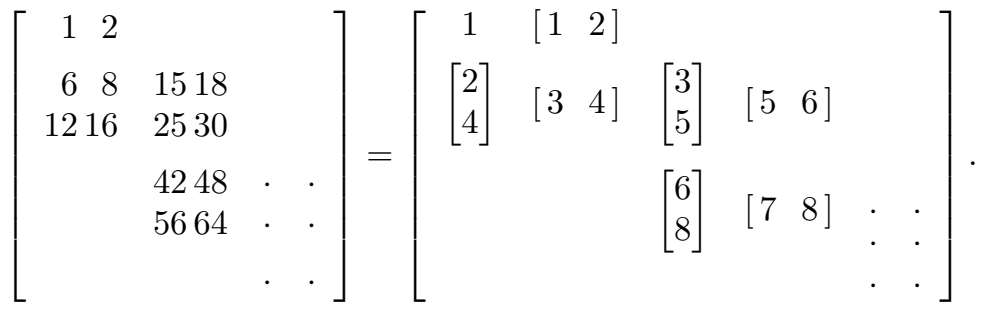

When a column of $B$ multiplies a row of $C$, the nonzeros sit in a 2 by 2 matrix of rank 1 . These 2 by 2 matrices do not overlap in $B C$. So when those column-row products are added (a legal way to compute $B C$ ), the result is a "CMV matrix." This matrix $B C$ has two diagonals of singular 2 by 2 blocks.

The inverse matrix $(B C)^{-1}=C^{-1} B^{-1}$ has a similar multiplication in the opposite order. The pattern of nonzeros is just the transpose of the pattern above. This product is another CMV matrix (singular 2 by 2 blocks side by side).

This paper will describe a corresponding factorization for other (smaller or larger) groups of matrices. Here are four of those groups, not an exhaustive list:

(1) Banded orthogonal matrices. The inverse is the transpose (so automatically banded). The factors $F$ will now be orthogonal matrices with $w=1$ : block diagonal with small orthogonal blocks.

(2) Banded permutation matrices. In this case $w=\max |i-p(i)|$ measures the maximum movement from $i$ in $(1, \ldots, n)$ to $p(i)$ in $(p(1), \ldots, p(n))$. Each factor $F$ is then a permutation with $w=1$; it exchanges disjoint pairs of neighbors.

We conjectured that fewer than $2 w$ factors $F$ would be sufficient. Greta Panova has found a beautiful proof [6]. Other constructions [1, 9] also yield $N \leq 2 w-1$.

(3) Banded plus finite rank. For infinite matrices $A$, banded with banded inverse, we enlarge to a group $B$ by including also $A+Z$. The perturbation $Z$ allows any matrix of finite rank such that $A+Z$ is invertible. Then its inverse will be $A^{-1}+Y$, also perturbed with $\operatorname{rank}(Y) \leq \operatorname{rank}(Z)$, and we have a group.

In this case, we include the new factors $F=I+($ rank 1$)$ along with the block diagonal $F$ 's. These factors generate the enlarged group. 
(4) Cyclically banded matrices. Cyclic means that " $n$ is adjacent to 1 ". The distance from diagonal $i$ to diagonal $j$ is the smaller of $|i-j|$ and $n-|i-j|$. The cyclic bandwidth of $A$ is the largest distance for which $A_{i j} \neq 0$. Then $w_{c}$ is the larger of the cyclic bandwidths of $A$ and $A^{-1}$.

The natural conjecture is $A=F_{1} \ldots F_{N}$ with factors that have cyclic bandwidth 1 (so that $F_{1 n}$ and $F_{n 1}$ may be nonzero). The number $N$ should be controlled by $w_{c}$. No proof of this conjecture is to be found in the present paper (but see [11] for a start).

\section{BANDED ORTHOGONAL MATRICES}

We need to recall how to obtain $A=B C$ with block diagonal factors $B$ and $C$. We construct orthogonal $B$ and $C$ when $A$ is orthogonal. Then the final step will separate $B$ and $C$ into orthogonal factors with $w=1$.

The key is to partition $A$ into blocks $H$ and $K$ of size $2 w$. Each of those blocks has rank exactly $w$. This comes from a theorem of Asplund about ranks of submatrices of $A$, when the inverse matrix has bandwidth $w$. The display shows a matrix for which $A$ and $A^{-1}$ have bandwidth at most $w=2$ :

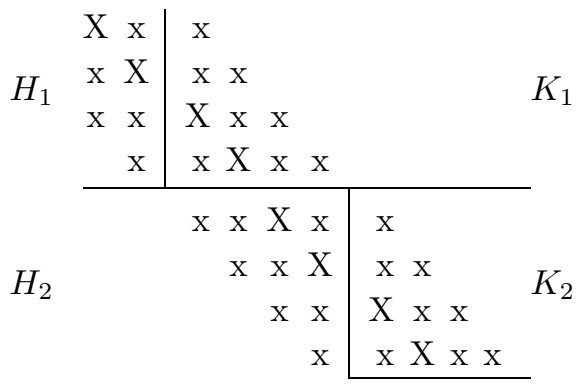

Asplund's theorem applies to submatrices such as the $K$ 's that are above the subdiagonal $w$, and like the $H$ 's that are below the superdiagonal $w$. "Those submatrices have rank $\leq w[2,11]$." In our case the ranks are exactly $w$ because each set of $2 w$ rows of $A$ must have full rank $2 w$ (since $A$ is invertible).

The two columns of $H_{1}$ are orthonormal. Choose a 4 by 4 orthogonal matrix $Q_{1}$, such that we get two columns correct in the identity matrix:

$$
Q_{1} H_{1}=\left[\begin{array}{ll}
1 & 0 \\
0 & 1 \\
0 & 0 \\
0 & 0
\end{array}\right]=R
$$

Since the four rows of $Q_{1}\left[\begin{array}{ll}H_{1} & K_{1}\end{array}\right]=\left[\begin{array}{ll}R & Q_{1} K_{1}\end{array}\right]$ are orthonormal, the first two rows of $Q_{1} K_{1}$ must be zero. Then there is a 4 by 4 orthogonal matrix $C_{1}{ }^{\mathrm{T}}$ acting on the columns of $Q_{1} K_{1}$ that produces $R^{\mathrm{T}}$ in the next two rows:

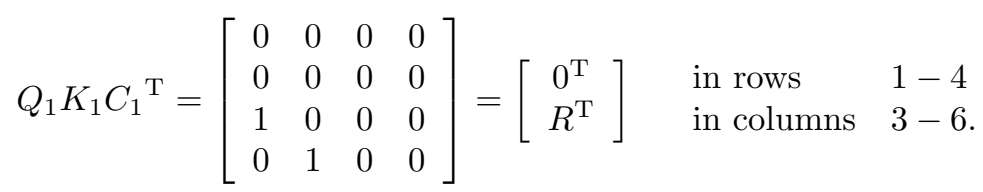

At this stage the first $2 w=4$ rows are set. $Q_{1} A C_{1}{ }^{\mathrm{T}}$ agrees with the first four rows of $I$. If $B_{1}=Q_{1}^{-1}$ is placed into the first block of $B$, and $C_{1}$ in rows/columns 5 to 8 of $C$, then four rows of $A=B C$ are now correct. 
Moving to the next four rows, our goal is to change those into four more rows of the identity matrix. This will put the 8 by 10 submatrix of $H$ 's and $K$ 's in its final form:

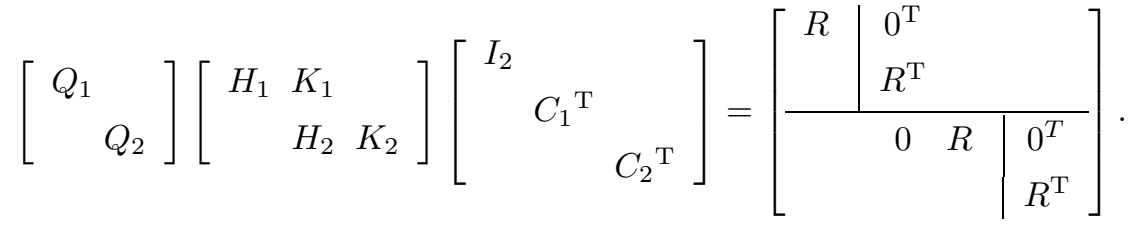

Columns 3 and 4 end with the zero matrix as indicated, because those columns are orthonormal and they already have 1's from $R^{\mathrm{T}}$.

Rows 5 to 8 are now in exactly the same situation that we originally met for rows 1 to 4 . There is an orthogonal matrix $Q_{2}$ that produces $R$ in columns 5 and 6 , as shown. The rest of rows 5 and 6 must contain $0^{\mathrm{T}}$, as shown. Then an orthogonal $C_{2}{ }^{\mathrm{T}}$ produces $R^{\mathrm{T}}$ in rows 7 and 8 . We now have eight rows of $Q A C^{\mathrm{T}}=I$. The construction continues to rows 9 to 12 , and onward:

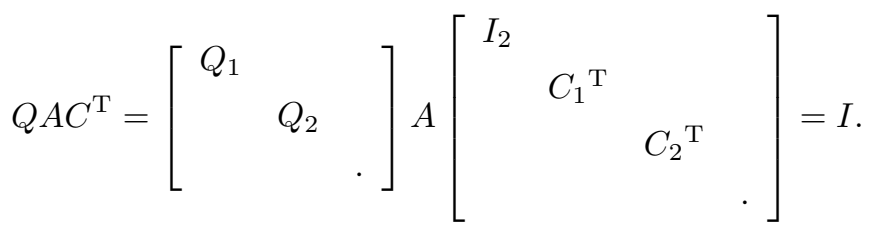

$Q$ and $C$ are block diagonal with orthogonal 4 by 4 blocks, except for the 2 by 2 block $I_{2}$. We have shown that every banded orthogonal matrix $A$ with $w=2$ can be factored into $A=Q^{\mathrm{T}} C=B C$. The reasoning is the same for any $w$.

Theorem 2.1. Every banded orthogonal matrix $A$, finite or singly infinite, has orthogonal block diagonal factors $A=Q^{-1} C=B C$. The blocks $B_{i}$ and $C_{i}$ have size $2 w$ except that $C_{0}=I$ has size $w$ (to produce the offset).

This completes the main step in the factorization: we have $B$ and $C$ with orthogonal blocks $B_{i}$ and $C_{i}$. The final step is to reach 2 by 2 blocks. A straightforward construction succeeds for each $B_{i}$ and $C_{i}$. At the end we factor all $B_{i}$ at once and all $C_{i}$ at once.

Lemma 2.2. Any orthogonal matrix $Q$ of size $2 w$ can be reduced to the identity matrix by a sequence of multiplications, $G_{M} \ldots G_{1} Q=I$. Each factor $G$ differs from $I$ only in a 2 by 2 orthogonal block (a plane rotation):

$$
G=\left[\begin{array}{cccc}
I_{m} & & & \\
& c & -s & \\
& s & c & \\
& & & I_{p}
\end{array}\right]
$$

Proof. Start at the bottom of column 1. Choose $c=\cos \theta$ and $s=\sin \theta$ to produce zero from $s Q_{n-1,1}+c Q_{n, 1}$ in that corner of $G_{1} Q$. (Take $s=1$ and $c=0$ in case $Q_{n-1,1}=0$.) Move up column 1, producing a new zero with each factor $G$. At the top of column 1, choose signs to produce 1 as the diagonal entry in this unit vector.

Continue from the bottom of column 2. The new factors $G$ that produce zeros below the diagonal will not affect the zeros in column 1 . The $(1,2)$ entry in column 2 
is already zero because the columns remain orthogonal at every step. At the end, we have the columns of $I$ from $M=O\left(w^{2}\right)$ orthogonal matrices $G_{i}$.

In the nonorthogonal case, elimination in this order (climbing up each column) yields $N=O\left(w^{2}\right)$ factors $F$ in Theorem 1.1. Each zero is produced by a "Gauss matrix" that has a single nonzero next to its diagonal part I. The $O\left(w^{3}\right)$ estimate in [10] was over-generous.

This lemma applies to each orthogonal block $B_{i}$ (of size $2 w$ ) in the matrix $B$. The key point is that we can simultaneously reduce all those blocks to $I$. The matrices $G_{1}, \ldots, G_{M}$ for all the different blocks of $B$ go along the diagonals of $F_{1}, \ldots, F_{M}$. Then $F_{M} \ldots F_{1} B=I$.

Similarly the lemma applies to the blocks $C_{i}$ of $C$. Then we have $A=B C=$ $\left(F_{1}^{-1} \ldots F_{M}^{-1}\right)\left(F_{M+1}^{-1} \ldots F_{2 M}^{-1}\right)$. This completes the orthogonal case.

\section{WAVELET MATRICES}

The matrices that lead to wavelets are banded with banded inverses. Furthermore they are block Toeplitz (away from the first and last rows) with 2 by 2 blocks. In this case the factors $F$ will also be block Toeplitz (away from those boundary rows). This means that each $F_{i}$ will have a 2 by 2 block repeating down the diagonal.

In the analysis of wavelets it is often convenient to work with doubly infinite matrices $A_{\infty}$ (purely block Toeplitz, with no boundary rows). Suppose $A_{\infty}$ and its inverse have bandwidth $w=N$, coming from $N$ blocks centered on each pair of rows. We showed in [10] how to find $N$ block diagonal factors in $A_{\infty}=F_{1} \ldots F_{N}$ : First find $N-1$ factors $G_{i}$, each with two singular 2 by 2 blocks per row $(w=2)$. Then split each $G_{i}$ into block diagonal factors $F_{i 1} F_{i 2}$. Those are offset as in equation (1.1) above, and we choose the factors so that $F_{i 2}$ is not offset compared to $F_{i+1,1}$. Then $F_{i 2} F_{i+1,1}$ is a block diagonal $F$, and the banded matrix $A_{\infty}$ has $N$ factors:

$$
\begin{aligned}
A_{\infty} & =\left(F_{11} F_{12}\right)\left(F_{21} F_{22}\right) \cdots\left(F_{N-1,1} F_{N-1,2}\right) \\
& =F_{11}\left(F_{12} F_{21}\right)\left(F_{22} F_{31}\right) \cdots\left(F_{N-2,2} F_{N-1,1}\right) F_{N-1,2} .
\end{aligned}
$$

The 4-coefficient Daubechies wavelet matrix has $N=2$. It is the single matrix $G_{1}$. It was factored in [5] into block diagonal $F_{11}$ times $F_{12}$ :

$$
\begin{gathered}
{\left[\begin{array}{ccc}
\bullet & 1+\sqrt{3} & -1+\sqrt{3} \\
& 1-\sqrt{3} & 1+\sqrt{3} \\
& & \bullet
\end{array}\right]\left[\begin{array}{cccc}
\sqrt{3} & -1 & & \\
1 & \sqrt{3} & & \\
& & \sqrt{3} & -1 \\
& 1 & \sqrt{3}
\end{array}\right]} \\
= \\
\left.\qquad \begin{array}{cccc}
\bullet & \bullet & & \\
1+\sqrt{3} & 3+\sqrt{3} & 3-\sqrt{3} & 1-\sqrt{3} \\
1-\sqrt{3} & -3+\sqrt{3} & 3+\sqrt{3} & -1-\sqrt{3} \\
& & \bullet & \bullet
\end{array}\right] .
\end{gathered}
$$

Again, column 2 times row 2 gives one singular block and column 3 times row 3 gives the other. Dividing by the lengths $\sqrt{8}$ and $\sqrt{4}$ of their rows, those factors become orthogonal matrices. Their product shows the $4+4$ Daubechies coefficients. The $6+6$ coefficient matrix for the next orthogonal Daubechies wavelet was factored by Raghavan 8]. 
These factors are plane rotations through $\pi / 12$ and $\pi / 6$. TeKolste also observed 13 that the Daubechies matrices are offset products of plane rotations. He recognized that the $N$ rotation angles add to $\pi / 4$. This connects to the fact that the coefficients in the second row of $A_{\infty}$ add to zero (a highpass filter). There should be additional conditions on the angles to determine the multiplicity of this zero in the highpass frequency response (the polynomial whose coefficients come from that second row of $A_{\infty}$ ).

A task for the future is to construct useful matrices $A_{\infty}$ and $A_{n}$ starting from well-chosen factors. Wavelets need not be orthogonal (the most popular choices are not), and they need not be restricted to block Toeplitz matrices. It seems feasible to construct time-varying wavelets by starting with factors $F$ in which the blocks are not constantly repeated down the diagonal. The matrix still has a banded inverse.

\section{BANDED PERMUtATion MATRICES}

The bandwidth of a permutation is the maximum distance $|i-p(i)|$ that any entry is moved. Thus $w=1$ for a "parallel exchange of neighbors" such as 2,1 , 4,3 . The matrix $F$ for this permutation is block diagonal with a 2 by 2 block for each exchange.

It is straightforward to reach the identity by a sequence of these $F$ 's. At each step we move from left to right, exchanging pairs of neighbors that are in the wrong order:

$456123 \rightarrow 451623 \rightarrow 415263 \rightarrow 142536 \rightarrow 124356 \rightarrow 123456$.

The original permutation has $w=3$ (in fact all entries have $|i-p(i)|=3$ ). Five steps were required to reach the identity. So the banded permutation matrix is the product of $N=5=2 w-1$ matrices $F$.

We conjectured in 10 that $N \leq 2 w-1$ in all cases. A beautiful proof is given by Greta Panova 6, using the "wiring diagram" to decide the sequence of exchanges in advance. A second proof 1 by Albert, Li, Strang, and Yu confirms that the algorithm illustrated above also has $N \leq 2 w-1$. A third proof is proposed by Samson and Ezerman 9.

\section{Finite RANK PERTURBATIONS}

We now consider the larger set of invertible matrices $M=A+Z$, when $A$ and $A^{-1}$ have bandwidth $\leq w$ and $Z$ has rank $\leq r$. The inverse matrix $M^{-1}$ has the same properties, from the Woodbury-Morrison formula (which has many discoverers). Write $Z$ as $-U V$ where $U$ has independent columns and $V$ has independent rows. Then the formula yields

$$
M^{-1}=(A-U V)^{-1}=A^{-1}+Y=A^{-1}+A^{-1} U\left(I-V A^{-1} U\right)^{-1} V A^{-1} .
$$

The rank of $Y$ is not greater than the ranks $($ both $\leq r)$ of $U$ and $V$.

The product $M_{1} M_{2}$ will be the sum of $A_{1} A_{2}$ with bandwidth $\leq 2 \mathrm{w}$, and $A_{1} Z_{2}+$ $A_{2} Z_{1}+Z_{1} Z_{2}$ with rank $\leq 3 r$. So we have a group $B$ of invertible matrices $M=$ $A+Z$, in which $A$ and $A^{-1}$ belong to our original group and rank $(Z)$ is finite. As before, $B$ is all of $\operatorname{GL}(n)$ for finite size $n$. It is apparently a new group for $n=\infty$.

We want to describe a set of generators for this group. They will include the same block diagonal factors $F$ with bandwidth 1 , together with new factors of the form $I+($ rank 1$)$. We show how to express $A^{-1} M=I+A^{-1} Z$ using at most $r$ of these new factors. 
Theorem 5.1. If $L=A^{-1} Z$ has rank $r$, there are vectors $u_{1}, v_{1}, \ldots, u_{r}, v_{r}$ so that

$$
I+L=\left(I+u_{r} v_{r}^{\mathrm{T}}\right) \ldots\left(I+u_{1} v_{1}^{\mathrm{T}}\right) .
$$

Proof. We will choose vectors such that $v_{i}^{\mathrm{T}} u_{j}=0$ for $i>j$. Then if the columns of $V$ and $U$ are $v_{1}, \ldots, v_{r}$ and $u_{1}, \ldots, u_{r}$, the product $V^{\mathrm{T}} U$ is upper triangular.

Under this condition, the expression (5.2) reduces to $I+u_{r} v_{r}^{\mathrm{T}}+\cdots+u_{1} v_{1}^{\mathrm{T}}=$ $I+U V^{\mathrm{T}}$. So the goal is to achieve $U V^{\mathrm{T}}=L$ with $V^{\mathrm{T}} U$ upper triangular.

The usual elimination steps reduce $L$ to a matrix $W$ with only $r$ nonzero rows. Thus $E L=W$ or $L=E^{-1} W=\left(E^{-1}\right)_{r} W_{r}$, where we keep only those first $r$ rows of $W$ and the first $r$ columns of $E^{-1}$. In the opposite order, $W_{r}\left(E^{-1}\right)_{r}$ may not be triangular, but every matrix is similar to an upper triangular matrix:

For some $r$ by $r$ matrix $S, \quad S W_{r}\left(E^{-1}\right)_{r} S^{-1}$ is upper triangular.

Now take $V^{\mathrm{T}}=S W_{r}$ and $U=\left(E^{-1}\right)_{r} S^{-1}$. Then $V^{\mathrm{T}} U$ is triangular and $U V^{\mathrm{T}}=L$.

\section{Cyclically banded matrices}

A cyclically banded $n$ by $n$ matrix $A$ (with bandwidth $w$ ) has three nonzero parts. There can be $w$ nonzero diagonals in the upper right corner and lower left corner, in addition to the $2 w+1$ diagonals in the main band. Call the new parts in the corners $b$ and $c$ :

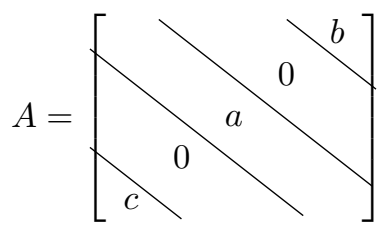

$$
\begin{aligned}
& A_{i j}=0 \text { if }|i-j|>w \\
& \text { and also } n-|i-j|>w .
\end{aligned}
$$

Thus the "cyclic bandwidth $w=1$ " now allows $A_{1 n} \neq 0$ and $A_{n 1} \neq 0$.

There is a corresponding infinite periodic matrix that has bandwidth $w$ in the normal way. "Periodic" means that $A_{i+n, j+n}=A_{i j}$ for all integers $i$ and $j$. When we identify 0 with $n$ and every $i$ with $n+i$, the corner parts $b$ and $c$ move to fill the gaps at the start and end of the main band. Here is an example with $b=4$ and $c=3$ and all 1's in the tridiagonal $a$ :

$$
\text { Periodic } A_{\infty}=\left[\begin{array}{rr|rrr|rr}
1 & 1 & 3 & & & & \\
\hline & 4 & 1 & 1 & & & \\
& & 1 & 1 & 1 & & \\
& & & 1 & 1 & 3 & \\
& & & & 4 & 1 & 1
\end{array}\right] \text { with cyclic } w=1 .
$$

Notice that the periodic matrix $A_{\infty}$ is block Toeplitz. The banded blocks $a$ repeat down the main diagonal, and blocks $b$ and $c$ (all 3 by 3 ) go down the adjacent diagonals. Multiplication $A B$ of cyclically banded matrices corresponds to $A_{\infty} B_{\infty}$ for periodic matrices. Inversion $A^{-1}$ corresponds to $\left(A_{\infty}\right)^{-1}$. The cyclic bandwidth for $A$ is the normal bandwidth for $A_{\infty}$.

The reader will make the same conjecture as the author:

Conjecture. If $A$ and $A^{-1}$ have cyclic bandwidth $\leq w$, they are products of $N=$ $O\left(w^{2}\right)$ factors for which $F$ and $F^{-1}$ have cyclic bandwidth $\leq 1$. The number $N$ does not depend on $n$. 
For cyclically banded permutation matrices, this conjecture is proved by Greta Panova. Her wiring diagrams move to a cylinder, for periodicity. The number of factors still satisfies $N \leq 2 w-1$ (for permutations). The parallel transpositions in a factor $F$ can include an exchange of 1 with $n$. Two new factors are allowed that also have cyclic bandwidth 1 ; these are the cyclic shifts. They permute $1, \ldots, n$ to $n, 1, \ldots, n-1$ or to $2, \ldots, n, 1$. The inverse of one cyclic shift is the other. The number of these additional factors is the "shifting index" of $P$ [11]. That index, positive or negative, satisfies $|s(P)| \leq w$.

Allow us to close the paper with preliminary comments on a proof of the conjecture. The noncyclic proof began with Asplund's condition on a matrix $A$ whose inverse has bandwidth $\leq w$. All submatrices of $A$ above the subdiagonal $w$ and all below the superdiagonal $w$ have rank $\leq w$. We expect this condition to extend to periodic infinite matrices $A_{\infty}$, but one change is important: The cyclic shift $S$ (and its periodic form $S_{\infty}$ ) is inverted by its transpose. The finite matrix obeys Asplund's rule:

$$
S=\left[\begin{array}{llll}
0 & 0 & 0 & 1 \\
1 & 0 & 0 & 0 \\
0 & 1 & 0 & 0 \\
0 & 0 & 1 & 0
\end{array}\right] \quad \begin{gathered}
\text { has rank } 1 \text { above subdiagonal 1: } \\
\text { then } S^{-1} \text { has upper bandwidth } 1 \\
\text { has rank } 3 \text { below superdiagonal 3: } \\
\text { then } S^{-1} \text { has lower bandwidth 3. }
\end{gathered}
$$

But $S_{\infty}$ needs a revised rule. It is lower triangular (rank zero above the diagonal), but its inverse (the transpose shift) is upper triangular.

The second obstacle, perhaps greater, lies in the factorization of $A_{\infty}$ into block diagonal periodic matrices $B_{\infty} C_{\infty}$. As in Section 2 above, their diagonal blocks may have size $2 w$ (independent of $n$ ). The further factorization into periodic matrices $F$ with 2 by 2 blocks would be straightforward, but how to reach $B_{\infty}$ and $C_{\infty}$ ? The elimination steps we used earlier are now looking for a place to start.

In our original proof of the (noncyclic) factorization into $A=F_{1} \ldots F_{N}$, a decomposition of "Bruhat type" was the first step in order to reach triangular factors:

$$
A=(\text { triangular }) \text { (permutation) (triangular) }=L P U .
$$

With $P$ factored into $F$ 's, this leaves the triangular $L$ and $U$ to be factored. $L, P, U$ are all banded with banded inverses; their bandwidths come from $A$ and $A^{-1}$. Note that Bruhat places $P$ between the triangular factors, where numerical linear algebra places it first. (The standard Bruhat triangular factors are both upper triangular.)

In the periodic case, $P_{\infty}$ will be an affine permutation. The banded periodic matrix $A_{\infty}$ is naturally associated with a rational matrix function $a(z)$. The blocks in $A_{\infty}$ are the coefficients in $a(z)$. For our block tridiagonal matrix, this function will be $b z^{-1}+a+c z$.

Then the triangular $L_{\infty} P_{\infty} U_{\infty}$ factorization of $A_{\infty}$ is associated with a factorization of this $n$ by $n$ matrix function, as in

$$
a(z)=l(z) p(z) u(z) \text { with } p(z)=\operatorname{diag}\left(z^{k_{1}}, \ldots, z^{k_{n}}\right) .
$$

The integers $k_{1}, \ldots, k_{n}$ are the (left) partial indices of $a(z)$. They determine the periodic permutation matrix $P_{\infty}$; the 1 in row $i$ lies in column $i+k_{i} n$. The factors $u(z)$ and $l(z)$ are analytic for $|z|<1$ and $|z|>1$. Their matrix coefficients appear as the blocks in the triangular factors $U_{\infty}$ and $L_{\infty}$. 
Matrix factorization theory began with the fundamental paper of Plemelj [7]. A remarkable bibliography and a particularly clear exposition and proof of (6.2) are in the survey paper [4] by Gohberg, Kaashoek, and Spitkovsky. They include a small example that we convert from $a(z)=l(z) p(z) u(z)$ to $A_{\infty}=L_{\infty} P_{\infty} U_{\infty}$ with $U_{\infty}=I$. Rows 1 to 4 show the pattern in these infinite block Toeplitz matrices:

$$
\begin{aligned}
& a(z)=\left[\begin{array}{cc}
z & 0 \\
1 & z^{-1}
\end{array}\right]=\left[\begin{array}{cc}
1 & 0 \\
z^{-1} & 1
\end{array}\right]\left[\begin{array}{ll}
z & \\
& z^{-1}
\end{array}\right]\left[\begin{array}{ll}
1 & 0 \\
0 & 1
\end{array}\right]=\operatorname{lp} u, \\
& A_{\infty}=\left[\begin{array}{llllllll}
0 & 0 & 0 & 0 & 1 & 0 & & \\
0 & 1 & 1 & 0 & 0 & 0 & \\
& & 0 & 0 & 0 & 0 & 1 & 0 \\
& & 0 & 1 & 1 & 0 & 0 & 0
\end{array}\right]=L_{\infty} P_{\infty} \\
& =\left[\begin{array}{llllllll}
0 & 0 & 1 & 0 & 0 & 0 & & \\
1 & 0 & 0 & 1 & 0 & 0 & & \\
& & 0 & 0 & 1 & 0 & 0 & 0 \\
& & 1 & 0 & 0 & 1 & 0 & 0
\end{array}\right]\left[\begin{array}{llllllll}
0 & 0 & 0 & 0 & 1 & 0 & & \\
0 & 1 & 0 & 0 & 0 & 0 & & \\
& & 0 & 0 & 0 & 0 & 1 & 0 \\
& & 0 & 1 & 0 & 0 & 0 & 0
\end{array}\right] \text {. }
\end{aligned}
$$

Important. The inverse of $A_{\infty}$ is also banded in this example because the determinant of $a(z)$ is a monomial. The inverse of this $L_{\infty}$ reverses signs below the diagonal, and the inverse of a permutation $P_{\infty}$ is always the transpose. With indices $k_{1}=1$ and $k_{2}=-1$ and $n=2$, rows 1 and 2 of $P_{\infty}$ have ones in columns $1+2=3$ and $2-2=0$.

Two key questions stand out:

(1) Block diagonal factorization of the periodic matrices $U_{\infty}$ and $L_{\infty}$.

(2) Nonperiodic infinite matrices $A$, banded with banded inverses: Do they factor into $F_{1} \ldots F_{N}$ (block diagonal and shifts)?

\section{REFERENCES}

1. C. Albert, C.-K. Li, G. Strang and G. Yu, Permutations as products of parallel transpositions, submitted to SIAM J. Discrete Math. (2010).

2. E. Asplund, Inverses of matrices $\left\{a_{i j}\right\}$ which satisfy $a_{i j}=0$ for $j>i+p$, Math. Scand. 7 (1959) 57-60. MR0109833 (22:718)

3. I. Daubechies, Orthonormal bases of compactly supported wavelets, Comm. Pure Appl. Math. 41 (1988) 909-996. MR 951745 (90m:42039)

4. I. Gohberg, M. Kaashoek, and I. Spitkovsky. An overview of matrix factorization theory and operator applications, Operator Th. Adv. Appl., Birkhäuser (2003) 1-102. MR2021095 (2004j:47034)

5. V. Olshevsky, P. Zhlobich, and G. Strang, Green's matrices, Lin. Alg. Appl. 432 (2010) 218241. MR2566471 (2011a:15017)

6. G. Panova, Factorization of banded permutations, arXiv.org/abs/1007.1760 (2010).

7. J. Plemelj, Riemannsche Funktionenscharen mit gegebener Monodromiegruppe, Monat. Math. Phys. 19 (1908) 211-245. MR.1547764

8. V.S.G. Raghavan, Banded Matrices with Banded Inverses, M.Sc. Thesis, MIT (2010).

9. M.D. Samson and M.F. Ezerman, Factoring permutation matrices into a product of tridiagonal matrices, arXiv.org/abs/1007.3467 (2010).

10. G. Strang, Fast transforms: Banded matrices with banded inverses, Proc. Natl. Acad. Sci. 107 (2010) 12413-12416. MR2670987

11. G. Strang, Banded matrices with banded inverses and A = LPU, Proceedings of ICCM2010 (International Congress of Chinese Mathematicians, Beijing, December 2010). 
12. G. Strang and T. Nguyen, The interplay of ranks of submatrices, SIAM Review 46 (2004) 637-646. MR2124679(2005m:15015)

13. K. TeKolste, private communication (2010).

Department of Mathematics, Massachusetts Institute of Technology, Cambridge, MASSACHUSETTS 02139

E-mail address: gs@math.mit.edu 